\title{
Combined Computational and Experimental Investigations on the Diastereoselective Hydrogenation of Steroids by Chiral Oxazaborolidine
}

\author{
GY. GÖNDÖS, I. PÁLINKÓ \\ Department of Organic Chemistry, University of Szeged, Dóm tér 8, Szeged, H-6720 Hungary
}

Received 4 September 2000; revised 28 September 2000; accepted 20 February 2001

\begin{abstract}
In this work the results of semiempirical quantum chemical calculations are described that support experimental findings applying the methyl derivative of oxazaborolidine- $\mathrm{BH}_{3}$ chiral complex for the reduction of a number of pregnane derivatives ( $3 \alpha$-hydroxy-5 $\beta$-pregnane-20-one, $3 \alpha, 17 \alpha$-dihydroxy-5 $\beta$-pregnane-20-one, $3 \alpha, 21$-dihydroxy- $5 \beta$-pregnane-20-one, and $3 \alpha, 17 \alpha, 21$-trihydroxy-5 $\beta$-pregnane-20-one). Calculations were performed by the MNDO method. The reactants, the products, the assumed transition states, as well as the assumed intermediate structures were optimized without any constraints. Calculations were able to reproduce the observed activity sequence and explain the experimental finding that the thermodynamically less stable isomer is formed preferentially. (c) 2001 John Wiley \& Sons, Inc. Int J Quantum Chem 84: 253-258, 2001
\end{abstract}

Key words: pregnane derivatives; semiempirical quantum chemical calculations; MNDO method; reduction; chiral methyl-oxazaborolidine

\section{Introduction}

$\mathbf{E}$ nantio- or diastereoselective reactions may be performed in both the homogeneoues and the heterogeneous phase [1]. The latter is more advantageous (either modified supported metals or

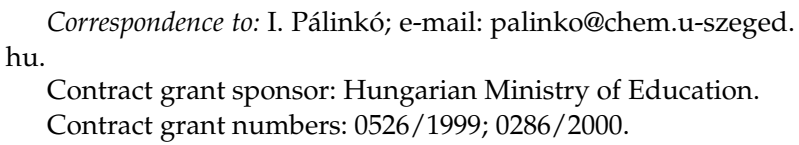

heterogenized organometallic complexes are used) in the economic point of view since the catalyst can be recycled; therefore, it is cheaper and also induces less pressure on the environment. However, unfortunately, most of the useful reactions do not proceed with high enough enantioselective excess over heterogeneous catalyts. Consequently, in most instances reactions in the homogeneous phase remains the only choice. Reactants and workup procedures are generally more expensive here, but costs may be reduced if the reactions can be made catalytic, and especially so if the catalyst can be recov- 
ered without extreme efforts. Therefore, discovering novel compounds capable of inducing enantio- or diastereoselective reactions and learning about their properties, especially about the factors that govern isomer distribution, are of great interest in both fundamental and practical points of view.

It was found some time ago that catalytic amounts of chiral oxazaborolidines were very effective in the diastereoselective reduction of relatively simple ketones [2]. It became clear soon that if a methyl substituent is on the boron, the material can be handled easier (it is less hygroscopic) and higher enantio- or diastereoselectivity can be achieved [3]. The large-scale synthesis of this compound has also been solved [4]. The relatively easy availability made possible its use for the diastereoselective reduction of more complicated compounds like steroids. The reduction of the keto function in position 20 to the $20 \alpha$ hydroxy isomer is relatively scarcely studied [5-7] and selectivity toward the $20 \alpha$ isomer was gener- ally low. High selectivity was only achieved when organoborohydrides and electrophilic organoboranes were used [8]. The possibility of applying catalytic amounts of oxazaborolidines for this reaction induced this study.

In this work we describe the results of semiempirical quantum chemical calculations that support experimental findings applying the methyl derivative of oxazaborolidine- $\mathrm{BH}_{3}$ chiral complex for the reduction of a number of pregnane derivatives.

\section{Methods of Evaluation}

The steroid ketones were $3 \alpha$-hydroxy- $5 \beta$-pregnane-20-one (1), $3 \alpha, 17 \alpha$-dihydroxy- $5 \beta$-pregnane-20one (2), 3 $\alpha, 21$-dihydroxy- $5 \beta$-pregnane-20-one (3), and $3 \alpha, 17 \alpha, 21$-trihydroxy-5 $\beta$-pregnane-20-one (4). The other reactant was the $\mathrm{BH}_{3}$ complex of the methyl derivative of oxazaborolidine (5). (The compounds are depicted in Figure 1.)<smiles>CC(=O)[C@]1(O)CCC2C3CC[C@H]4C[C@@H](O)CCC4(C)C3CCC21C</smiles><smiles>CC12CC[C@@H](O)C[C@H]1CCC1C2CCC2(C)C1CC[C@@]2(O)C(=O)CO</smiles><smiles>CB1BN2CCCC2CO1</smiles>

5

FIGURE 1. The reactants in this study. 
The reactions were performed at gradually increasing temperature starting from $203 \mathrm{~K}$ and finishing with 3-h reflux at $313-323 \mathrm{~K}$. The molecules were isolated and purified by column chromatography. The ratio of diastereomers were determined by high-pressure liquid chromatography. Details of synthesis and workup procedure is detailed in a follow-up study [9].

Calculations were performed at the level of semiempirical quantum chemistry by the MNDO method [10] included in the Hyperchem package [11]. The reactants, the products, the assumed transition states [3,12] as well as the assumed intermediate structures were optimized without any constraints. Energy (more precisely, enthalpies of formation) values were compared to each other and predictions were confronted with experimental results.

\section{Results and Discussion}

The experimental data are displayed in Table I. These results show that the introduction of an $\mathrm{OH}$ group to position 17 results in an appreciable decrease in conversion (for compound 4 reduction did not proceed at all). Comparing selectivity for compounds 1 and 2, the $20 \alpha$ to $20 \beta$ ratio also decreased.

Introducing $\mathrm{OH}$ substituent into position $21 \mathrm{ex}$ erts similar effects on both the conversion and the $20 \alpha$ to $20 \beta$ ratio (compare the specific values for compound 1 to those of compound 3). However, they are not so significant as for the substitution in position 17.

Computational modeling is performed to obtain reasonable explanation for the observed changes in conversion and selectivity values depending on the position of the $\mathrm{OH}$ group. The complete lack of reduction when $\mathrm{OH}$ substitution occurred in positions 17 and 21 (compound 4) especially should be interpreted.
TABLE I

Experimental yields and stereoselectivities in the reduction of steroid 20 ketones catalyzed by boroxazolidine- $\mathrm{BH}_{3}$ complex.

\begin{tabular}{ccc}
\hline Steroid ketone & $20 \alpha / 20 \beta$ & Yield (\%) \\
\hline $\mathbf{1}$ & $91: 9$ & 100 \\
$\mathbf{2}$ & $75: 25$ & 40 \\
$\mathbf{3}$ & $80: 20$ & 85 \\
$\mathbf{4}$ & - & - \\
\hline
\end{tabular}

On the basis of previously published suggestions, the main steps in the reduction mechanism may be summarized as follows [3, 12]:

1. The $\mathrm{BH}_{3}$ forms a complex with the boroxazolidine.

2. The ketone is coordinated to the atomic orbital of the boron in the boroxazolidine.

3. Hydride ion transfer takes place from the $\mathrm{NBH}_{3}{ }^{-}$unit to the carbonyl carbon.

4. Via hydrolysis the alcohol is formed.

A schematic representation is given in Scheme 1.

Enthalpies of formation data calculated by the MNDO method for the reactants, the products, the assumed transition states [12], and the assumed intermediates are summarized in Table II.

The assumed transformation pathway for compound 2 and the mechanism that can be envisioned for compound 4 (recall, actually this compound is unreactive in this transformation), as examples are shown in Schemes 2 and 3. In both schemes each structures is optimized by the MNDO method without constraints.

From the data in the Table II it is immediately seen that (i) the introduction of $\mathrm{OH}$ to position 17 exerts stabilizing effect on the reactants and (ii) the $20 \beta$ isomer products are more stable ther-<smiles>[R]C1O[B]N2CCC[C@]2([R])C1[R]</smiles><smiles>[R]C([R])=O</smiles>

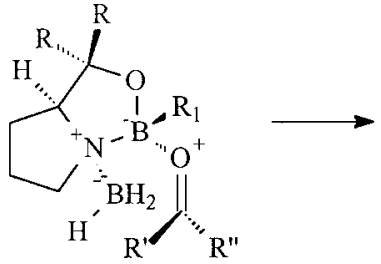

transition state

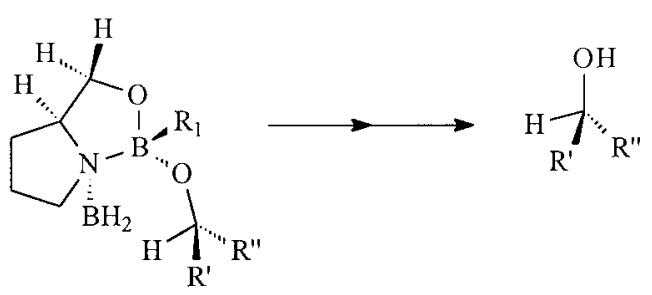

intermediate

SCHEME 1. Transformation pathway for the reduction of ketones by chiral oxazaborlidines [12]. 
TABLE II

Calculated (MNDO method) enthalpy of formation data (in $\mathrm{kcal} / \mathrm{mol}$ ) for the reactants, the assumed transition states (energy level differences from the reactants are in parentheses), the assumed intermediates, and the products in the reduction of the carbonyl function at position $\mathbf{2 0}$ of pregnane derivatives.

\begin{tabular}{lccccc}
\hline Reactant & Transition state & $\operatorname{Int}(20 \alpha)$ & $\operatorname{Int}(20 \beta)$ & $20 \alpha$ & $20 \beta$ \\
\hline 1: 63.75 & $86.40(22.65)$ & 82.92 & 79.88 & 68.11 & 63.75 \\
2: 59.24 & $92.49(33.25)$ & 87.01 & 82.21 & 60.7 & 56.89 \\
3: 68.73 & $92.05(23.32)$ & 82.88 & 80.21 & 69.35 & 66.41 \\
4: 57.17 & $95.50(38.33)$ & 94.20 & 82.34 & 62.09 & 58.10 \\
\hline
\end{tabular}

modynamically than the $20 \alpha$ isomer products. From this latter fact it follows that the observed isomer distribution cannot be explained by the thermodynamic stability difference in the products. It is also clear that the intermediates on the way to the $20 \beta$ isomers are also more stable than those leading to the $20 \alpha$ isomers. A somewhat closer look tells that the difference in the energy levels between the reactant and the transition state in the first step (reaching the intermediate states) is the highest for compound 4 . This is in agreement with the experimental observation that reduction is the most difficult for this compound, actually the reaction does not proceed. Even the reactivity sequence

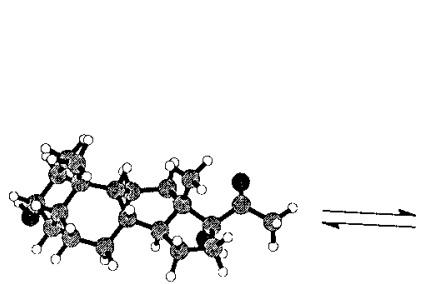

2

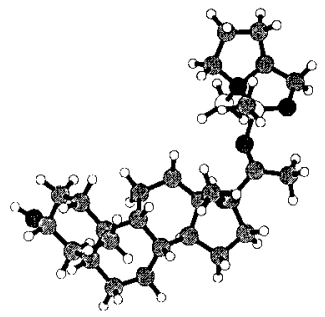

transition state

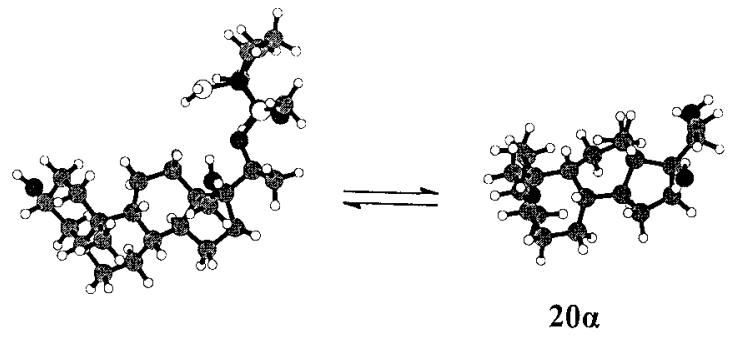

intermediate to $20 \alpha$
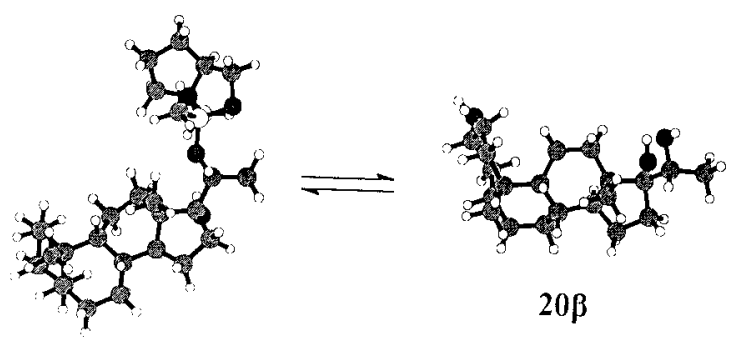

$20 \beta$

intermediate to $20 \beta$

SCHEME 2. Transformation routes in the reduction of compound 2 ( $3 \alpha, 17 \alpha$-dihydroxy-5 $\beta$-pregnane-20-one) at position 20 (full geometry optimizations of the reactant, the transition state, the intermediates, and the products were performed by the MNDO method; color code: large circles in decreasing darkness are O, C, B; small circle; H). 


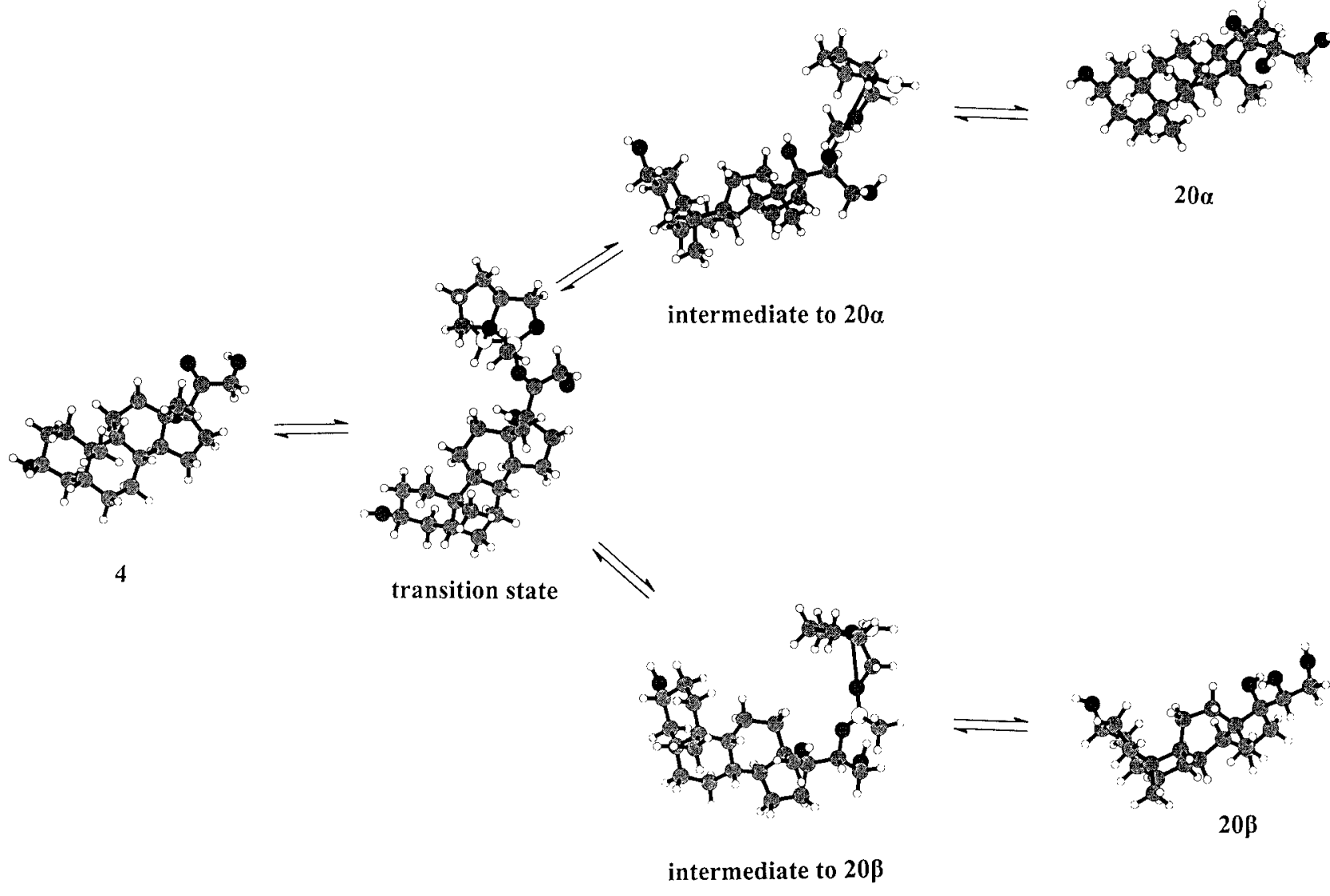

SCHEME 3. Imaginary transformation routes in the reduction of compound $4(3 \alpha, 11 \beta, 17 \alpha, 21$-tetrahydroxy-5 $\beta$ pregnane-20-one) at position 20 (full geometry optimizations of the reactant, the transition state, the intermediates, and the products were performed by the MNDO method; color code: large circles in decreasing darkness are O, C, B; small circle, $\mathrm{H})$.

expected on the basis of the computed enthalpies of formation for the assumed transition states belonging to compounds $1-3$ fits to the experiments, i.e., compound $\mathbf{1}$ is the most active, and activity decrease is more appreciable for compound 2 then for compound 3.

The alcohols are formed from the intermediates via hydrolysis and the products are more stable thermodynamically than the intermediates (thus, this step of the reaction is exothermic). Applying Hammond's postulate [13] and assuming that the energy levels of the transition complexes of the hydrolysis step are similar, it is to be expected that formation of the $20 \alpha$ isomer is favored. The energy level of this intermediate (Int20 $\alpha$ ) leading to the $20 \alpha$ isomer is closer to that of the transition complex (the structure of which resembles that of the intermediate). Indeed, this was found for each reactant, which undergoes transformation at all.

\section{Conclusions}

Comparison of the energy levels of the reactants, transition states, intermediates, and products in the diastereoselective reduction of pregnane 20-one derivatives [differing in the position(s) of $\mathrm{OH}$ substitution] could be used to explain the experimentally observed activity sequence and the unusual selectivity, i.e., the formation of the thermodynamically less favored isomer.

\section{ACKNOWLEDGMENTS}

This work was supported by the Hungarian Ministry of Education via grants 0526/1999 and $0286 / 2000$. The financial help is highly appreciated. 


\section{References}

1. Baiker, A. J Mol Catal A 1997, 115, 473-493 (and appropriate references therein).

2. Corey, E. J.; Bakshi, R. K.; Shibata, S. J Am Chem Soc 1987, 109, 5551-5553.

3. Corey, E. J.; Bakshi, R. K.; Shibata, S.; Chen, C.-P.; Singh, V. K. J Am Chem Soc 1987, 109, 7922-7926.

4. Mathre, D. J.; Jones, T. K.; Xavier, L. C.; Blacklock, T. J.; Reamer, R. A.; Mohan, J. J.; Jones, T. T.; Hoogsteen, K.; Baum, M. W.; Grabowski, E. J. J. J Org Chem 1991, 56, 751-762.

5. Göndös, Gy.; Orr, J. C. J Chem Soc Chem Commun 1982, $1238-1239$.
6. Göndös, Gy.; Gera, L.; Bartók, M.; Orr, J. C. J Organomet Chem 1989, 373, 365-375.

7. Göndös, Gy.; Orr, J. C. Liebigs Ann Chem 1990, 213-215.

8. Midland, M. M.; Kwon, Y. C. J Am Chem Soc 1983, 105, 3725-3727.

9. Göndös, Gy., to be submitted.

10. Dewar, M. J. S.; Thiel, W. J Am Chem Soc 1977, 99, 48994907; Dewar, M. J. S.; Thiel, W. J Am Chem Soc 1977, 99, 4907-4922.

11. Hyperchem 4.5; Hypercube: Gainesville, FL, 1994.

12. Jones, D. K.; Liotta, D. C.; Shinkai, I.; Mathre, D. J. J Org Chem 1993, 58, 799-801.

13. Hammond, G. S. J Am Chem Soc 1955, 77, 334-338. 